\section{Consummatory behavior as a function of deprivation level of the rat}

\author{
PETER M. MONTI* \\ College of William and Mary, Williamsburg, Va. 23185
}

The present investigation studied the relationship between strength of hunger drive and eating behavior. The experiment reexamined the usefulness of the Hullian concept of drive as opposed to an alternative position of stimulus generalization. Thirty-eight female albino rats were trained at $23 \mathrm{~h}$ of food deprivation and then tested at different deprivation intervals. Significant findings, showing an increase in eating behavior up to training level and a decrease thereafter, clearly support an associative interpretation of stimulus generalization as opposed to a strict drive position. Results are discussed in terms of the "associative" theory of Estes (1958). Alternative mechanisms are suggested in terms of digestive mechanisms and cyclical functions in the rat.

The present study is concemed with the influence of strength of hunger upon a strong response tendency, namely consummatory behavior. The major problem is to investigate the manner in which this measure of behavior changes as a function of the strength of the hunger drive at the time of testing.

Although drive strength as such is unobservable, it may be clearly inferred from certain antecedent conditions, such as length of interval during which an animal has been deprived of food or maintenance schedule characteristics, or from measures of subsequent behavioral events, such as eating, maze running, leverpressing, panel pushing, etc. (Horenstein, 1951). In the original formulation of Hull's (1943) theory, it was assumed that drive varied as an increasing function of the length of deprivation interval. The relationship between performance of a response and strength of hunger drive, at a given level of habit strength, is a function showing "slight positive acceleration [Hull, 1943, p. 228]." As Horenstein (1951) notes, the gradient upon which Hull's statements are based is determined empirically, producing a smooth and continuous curve.

In a series of three experiments, Horenstein (1951) examined the relationship between strength of hunger drive (as inferred from number of hours of food deprivation) and three measures of response strength. She found a sharp increase in response strength as deprivation period increased from 0 to $2 \mathrm{~h}$ and that, between 2 and $23^{1 / 2} \mathrm{~h}$ of deprivation, response strength increased more gradually.

Clearly, then, by manipulating deprivation intervals, response strength can be increased. However, as Brown

* Now at the University of Rhode Island.
(1961) points out, the potential utility of Horenstein's study as an estimator of drive (D) in Hull's sense of the term may be somewhat questionable because stimulus similarity is one of the variables believed to determine $h$ abit strength, which is unduly assumed to be constant. This variable might have affected Horenstein's results by way of deprivation-induced stimuli, as discussed by Brown. Within Hull's system, deprivation generates not only $\mathrm{D}$, the nondirective catalyst, but also, in most cases, distinctive internal stimuli $\left(\mathbf{S}_{\mathbf{D}}\right)$.

According to Horenstein (1951), the tendency to eat may have become more strongly associated with internal stimuli characteristic of a $23^{1 / 2}-\mathrm{h}$ deprivation period than with cues arising from shorter deprivation times. Clearly, then, the fact that Horenstein's curve was highest at the $23^{1 / 2}-\mathrm{h}$ point and lower at increasingly shorter deprivation intervais might be due in part to differences in associative strengths (Brown, 1961).

This sort of nonmotivational interpretation of data has been adopted by Pastman (1953), who suggests that, since the manipulation of certain so-called motivational variables may affect drive stimuli as well as $D$, it might be possible, and certainly more economical, to dispense with the concept of $D$ entirely and base interpretations of motivated stimuli.

Similarly, Estes's (1958) theory of motivational variables predicts that the probability that a response will occur depends upon the proportion of stimulus elements that have been conditioned to the response. In the present study, the internal drive stimuli assume a role of relatively great importance. From certain assumptions concerning increases in deprivation time and the relative weights of conditioned cues and extraneous cues, behavior solely upon changes in drive
Estes's theory predicts that if training is carried out at a single level of deprivation, asymptotic performance will be higher with more severe deprivation. Another prediction is that if training is carried out under a moderate level of deprivation, performance should become worse if deprivation is suddenly enhanced.

The present study investigates the problem which has risen out of the above conflicting interpretations. The hypothesis tested is not new. It has been examined by Birch, Burnstein, \& Clark (1958), who present a model for motivation in which drive is not a monotonically increasing function of hours of deprivation, but rises to a maximum at or beyond the time of deprivation employed in the maintenance schedule and then decreases with increased deprivation. These authors, however, used immature rats of mixed sexes in addition to having somewhat removed indicant responses-namely, food-trough depressions and running speed. Therefore, the present investigation seemed warranted.

It was reasoned that if $S s$ were food deprived on 23-h schedules and then fed at different intervals before and after scheduled deprivation intervals, the effect of "drive" or generalization, as the case may be, could be further investigated. Clearly, it would be expected, according to Hullian drive theory as well as "common sense," that the longer Ss went without food, up to certain limits, the hungrier they would be and, thus, the more they should eat. Alternatively, an associative or generalization position would predict that Ss' consummatory behavior would generalize from the interval at which they were trained, thereby suggesting that Ss should eat less as the test trials were further removed from the training interval. METHOD

The Ss were 38 naive female albino DUB/SDD rats (Flow Labs, Dublin, Va.), approximately 87 days old. They weighed 201-237 g.

The Ss were housed randomly in individual cages and were given 7 days to become acclimated to eating from foodcups. During this acclimation period, Ss were handled frequently and carried about the laboratory. All Ss became very tame and showed no apparent emotional responses to handling. Laboratory temperature remained at $76^{\circ} \mathrm{F}$ throughout the experiment, and lighting was continuous.

All Ss were placed on 23-h feeding schedules 18 days prior to the actual experiment. Daily feeding took place in the individual cages and consisted of $1 \mathrm{~h}$ of free eating from a foodcup of ground Purina Lab Chow Checkers. 


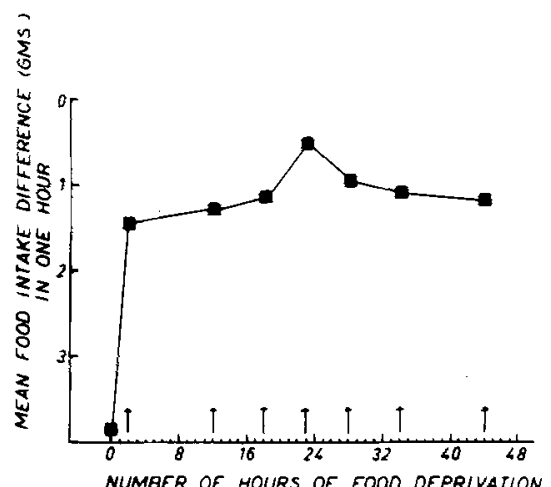

Fig. 1. Difference between mean food intake in $1 \mathrm{hr}$ for 3 days prior to the test day and test trial consumption as a function of number of hours of food deprivation.

Foodcups were weighed before and after each feeding period. This measure served as the indicant response. Body weight also was recorded after each feeding. Stale tap water was available during the feeding period as well as during the entire deprivation period.

At the end of the 18-day training period, when Ss' food intake had stabilized, Ss were assigned randomly to one of eight groups which were formed according to deprivation intervals of $0,2,12,18,23,28,34$, or $44 \mathrm{~h}$. Each group contained five $S$ except the 0 -h (control) group, which contained only three.

Tests under the various deprivation conditions were made on Days 18-20. After Ss had gone without food for a predetermined length of time (deprivation interval), foodcups were placed into their cages for $1 \mathrm{~h}$. This hour of free eating served as the test period. Test periods were scheduled according to the groups to which Ss had been assigned.

\section{RESULTS}

Although mean food intake among the groups for the 3 days prior to the test day was not statistically significant $(F=1.18, \mathrm{df}=7 / 30)$, there was, nonetheless, some variation. In order to control for individual differences within groups, a single treatment analysis was done using the differences between mean food intake for 3 days prior to the test day and test trial consumption ( $F=13.88$, $\mathrm{df}=7 / 30, \mathrm{p}<.01)$. These data are presented in Fig. 1. A $t$ test for carrying out multiple comparisons among means was performed on each mean difference score. The results of these comparisons are presented in Table 1.

The Ss regained $96 \%$ of their preexperimental body weight during the 20-day course of the experiment. An analysis of the variance of the regained body weight between groups was not found to be significant $(F<1$, $\mathrm{df}=7 / 30)$.

\section{DISCUSSION}

The results of the present experiment clearly confirm the observations of previous studies (e.g., Horenstein, 1951) that there is a sharp rise in measures of strength of response tendencies for periods of food deprivation between 0 and $2 \mathrm{~h}$. The finding that each response measure taken between 2 and $23 \mathrm{~h}$ of food deprivation shows a more gradual increase is consistent with the results of Horenstein (1951) and Perin (1942). Confirmation of previous findings is especially significant because the experimental techniques employed here were different from those employed in the studies cited. It is also interesting to note that, up until the $23-\mathrm{h}$ interval, the general shape of the curve obtained agrees rather nicely with Hull's formulation of primary drive. However, the most interesting finding occurs with those groups of animals that were tested on increasingly longer intervals than the 23-h interval at which they had been trained. The generalization curve obtained would clearly not be predicted by most of the previously cited studies and certainly not by Hull's formulation of primary drive. The results indicate a process of stimulus generalization that can be handled either by Birch, Burnstein, \& Clark's (1958) model for motivation or by Estes's (1958) associative conceptualization of stimulus generalization and drive-stimulus properties.

It should be recognized that a simple generalization around the intensity of drive stimulation employed in training, however, does not account for the sharp rise in the data curve between 0 and $2 \mathrm{~h}$ of deprivation. Clearly, there is another specific factor operative around the second hour of deprivation. Horenstein (1951) suggests that this 2-h factor may be physiological or strictly behavioral in nature. She notes the $2-3 \mathrm{~h}$ regular eating rhythm in the adult rat under free feeding conditions in addition to its similar pattern of activity, both of which make the consideration of a specific 2- or 3-h centered factor quite plausible

Further evidence of relevant physiological considerations is suggested in the studies of Reid \& Finger $(1955,1957)$. Empirically these show that rats require a considerable period of time to adjust their food intake to a new schedule and that the type of scheduling of eating periods results in differential efficiency in the digestion and use of food material. Mayer (1952) has reported evidence suggesting that along with changes in eating behavior, there are corresponding changes in the expenditure of energy through activity, heat loss, and the like. Clearly, if such physiological changes take place, then complete compensatory eating should not occur. since changes in the expenditure of energy will reduce the need for food.

Finally, Lawrence \& Mason (1955) have demonstrated that, for a given deprivation interval, Ss will eat more if the feeding period comes at a time when Ss are accustomed to eating than if it comes at some unfamiliar time. The present data would certainly support such a position, especially since the $44^{-h}$ interval group had a slight increase in mean food intake over the 34-h group, thereby suggesting that a group at a $46-\mathrm{h}$ interval may very well prove to regain maximum intake.

\section{REFERENCES}

BIRCH, D., BURNSTEIN, E., \& CLARK, R. A. Response strength as a function of hours of food deprivation under a controlled maintenance schedule. Journal of Comparative \& Physiological Psychology, 1958, 51, 350-354.

BROWN, J. S. The motivation of behavior New York: McGraw-Hill, 1961 .

ESTES, W. K. Stimulus-response theory of drive. In M. R. Jones (Ed.), Nebraska symposium on motivation. Lincoln: University of Nebraska Press, 1958 Po. 35-69.

HORENSTEIN, B. R. Performance of conditioned responses as a function of strength of hunger drive. Journal of Comparative \& Physiological Psychology, 1951, 44, 210-224.

HULL, C. L. Principles of behavior. New York: Appleton-Century-Crof ts, 1943.

LAWRENCE, D. H., \& MASON, W. A. Food intake in the rat as a function of deprivation intervals and rhythms. Journal of Comparative \& Physiological Psychology, 1955, 48, 267-271.

MAYER, J. The glucostatic theory of regulation of food intake and the

Table 1

t Test Comparing Differences Between Means

\begin{tabular}{ccccc}
\hline Comparisons & $x_{1}$ & $x_{2}$ & df & $t$ \\
\hline 0-h Group vs 23-h Group & 3.87 & .50 & 30 & $9^{* *}$ \\
2-h Group vs 23-h Group & 1.45 & .50 & 30 & $3^{* *}$ \\
12-h Group vs 23-h Group & 1.27 & .50 & 30 & $2.4^{*}$ \\
18-h Group vs 23-h Group & 1.11 & .50 & 30 & $1.9^{*}$ \\
28-h Group vs 23-h Group & .93 & .50 & 30 & 1.3 \\
34-h Group vs 23-h Group & 1.07 & .50 & 30 & $2.1^{*}$ \\
44-h Group vs 23-h Group & 1.14 & .50 & 30 & $2.0^{*}$ \\
\hline
\end{tabular}

$* p<.05, * * p<.01$ 
problem of obesity. Bulletin of North East Medical Center, 1952, 14, 43-49.

PERIN, C. T. Behavior potentiality as a joint function of the amount of training and the degree of hunger at the time of

extinction. Journal of Experimental Psychology, 1942, 30, 95-109.

POSTMAN, L. Comments on papers by
Professors Brown and Harlow. In M. R. Jones (Ed.), Current theory and research in motivation: A symposium. Lincoln: University of Nebraska Press, 1953. Pp. 55-58.

REID, L. S. \& FINGER, F, W. The rat's adjustment of 23-hour food deprivation cycles. Journal of Comparative \&
Physiological Psychology, 1955, 48. 110-113.

REID, L. S., \& FINGER, F. W. The effect of activity restriction upon adjustment to cyclic food deprivation. Journal of Comparative \& Physiological Psychology, $1957,50,491-494$.

\section{CURRENT LITERATURE ON ANIMAL MOTIVATION}

BRACKBILL, R. M. (Franklin and Marshall College, Lancaster, $\mathrm{Pa}$. 17604), \& BROOKSHIRE, K. H. Conditioned taste aversions as a function of the number of CS-US pairs. Psychonomic Science, 1971, 22, 25-26.

CROW, L. T. (Western Washington State College, Bellingham, Wash. 98225). Alcohol ingestion in rats following median eminence lesions. Psychonomic Science, 1971, 22, 36-37.

GROSSMAN, S. P. (University of Chicago, Chicago, Ill. 60637), \& GROSSMAN, L. Food and water intake in rats with parasagittal knife cuts medial or lateral to the lateral hy pothalamus. Journal of Comparative and Physiological Psychology, 1971, 74, 148-156.

HOWARD, R. L., \& MEYER, D. R. (202 Kinear Research Center, Ohio State University, Columbus, Ohio 43212). Motivational control of retrograde amnesia in rats: $A$ replication and extension. Journal of Comparative and Physiological Psychology, 1971, 74, 37-40.

LEE, C. T. (University of Texas, Austin, Tex. 78712), \& ESTEP, D. The developmental aspect of marking and nesting behaviors in
Mongolian gerbils (Meriones unguiculatus). Psychonomic Science, 1971, 22, 312-313.

OWNINGS, D. H. (University of Washington, Seattle, Wash. 98105), \& LOCKARD, R. B. Different nocturnal activity patterns of Peromyscus californicus and Peromyscus eremicus in lunar lighting. Psychonomic Science, $1971,22,63-64$.

PECK, J. W., \& NOVIN, D. (University of California, Los Angeles, Calif. 90024 ). Evidence that osmoreceptors mediating drinking in rabbits are in the lateral preoptic area. Journal of Comparative and Physiological Psychology, 1971, 74, 134-147.

PORTER, J. J. (University of Wisconsin-Milwaukee, Milwaukee, Wis. 53201), MADISON, H. L., \& SWATEK, A. J. Incentive and frustration effect of direct goal placements. Psychonomic Science, 1971, 22, 314-316.

SIDEROFF, S., SCHNEIDERMAN, N. (University of Miami, Coral Gables, Fla. 33124), \& POWELL, D. A. Motivational properties of septal stimulation as the US in classical conditioning of heart rate in rabbits. Journal of Comparative and
Physiological Psychology, 1971, 74, 1-10.

TANG, M., \& COLLIER, G. (RUTGERS-The State University, New Brunswick, N.J. 08903). Effect of successive deprivations and recoveries on the level of instrumental performance in the rat. Journal of Comparative and Physiological Psychology, 1971, 74, 108-114.

THOMPSON, R. D. (U.S. Bureau of Sport Fisheries and Wildlife, Denver Federal Center, Denver, Colo. 80225 ), \& GRANT, C. V. Automated preference testing apparatus for rating palatability of foods. Journal of the Experimental Analysis of Behavior, 1971, 15, 215-220.

WRIGHT, W. E. (George Peabody College, Nashville, Tenn. 37203), FOSHEE, D. P., \& McCLEARY, G. E. Comparison of taste aversion with various delays and Cyclophosphamide dose levels. Psychonomic Science, 1971, 22, 55-56. 